\title{
PSMC3 is required for spermatogonia niche establishment in mouse
}

\section{spermatogenesis}

Maria Cristina S. Pranchevicius ${ }^{1 \#}$, Luciana Previato ${ }^{2 \#}$, Rodrigo O. de Castro ${ }^{2}$, and Roberto J.

Key words: gametogenesis, spermatogonia, spermatogenesis, and testis development.

${ }^{1}$ Department of Genetics and Evolution, Universidade Federal de Sao Carlos, Sao Carlos, Brazil.

${ }^{2}$ Cell Cycle and Cancer Biology Research Program, Oklahoma Medical Research Foundation, Oklahoma City, Oklahoma, United States of America.

${ }^{3}$ Department of Cell Biology, University of Oklahoma Health Science Center, Oklahoma City, Oklahoma, United States of America.

\#First authors.

* To whom correspondence should be submitted.

Running title: Psmc3 function in mouse gametogenesis

Key words: gametogenesis, spermatogonia, spermatogenesis, and testis development.

\begin{abstract}
Males produce millions of spermatozoa each day, which are originated from spermatogonia. Spermatogonia niche establishment and maintenance and the subsequent haploidization of spermatocytes in meiosis are hallmarks of this process. The function of the individual players and coordinated mechanisms regulating different stages of gametogenesis in mammals are not well understood. In this work we focused on the role of PSMC3 in mouse gametogenesis. We observed that Psmc3 is highly expressed in mouse testis, and it is widely expressed in different
\end{abstract}


stages of gamete formation. Conditional deletion of Psmc3 results in both male and female impairment of gonad development at early pre-meiotic stages, but has no apparent effect on meiosis progression. This is likely a consequence of abnormal spermatogonia niche establishment and/or maintenance, revealed by a massive loss of undifferentiated spermatogonia. Our work defines a fundamental role of PSMC3 functions in spermatogenesis during spermatogonia development with direct implications in fertility.

\section{Introduction}

Spermatogenesis is the process by which diploid spermatogonia cells produce haploid mature gametes called spermatozoa. During this process, spermatogonia niche establishment and maintenance are essential to ensure that the genetic information is passed to the next generation. Indeed, errors in producing or differentiating spermatogonia can result in male infertility. Spermatogenesis begins with primordial germ cells migration to the developing male gonad and division to produce an undifferentiated stem cell type of spermatogonia (type A). The latter cells divide and some of them generate differentiated type B spermatogonia, the last to undergo mitotic division and the precursor of primary spermatocytes. Primary spermatocytes undergo meiosis to half their chromosome complement and yield a pair of secondary spermatocytes, which differentiate to produce haploid gametes [1].

In order to explain possible causes of infertility, we need to identify and understand the function of the individual players and coordinated mechanisms regulating mammalian spermatogenesis. Here, we focus on understanding the role of PSMC3 in mouse germ cell development. Analysis of mouse PSMC3 (a.k.a. TBP1/Tat-binding protein 1, RPT-5 or S6A) protein sequence indicates that PSMC3 belongs to the AAA ATPase family of proteins. Starting from the amino-terminus, this protein features a putative leucine zipper motif with possible DNA 
binding activity; an ATPase Walker A motif, an ATPase Walker B motif; and a putative helicase domain with a DEXD motif, that relates PSMC3 to the superfamily 2 DEAH helicases.

PSMC3 has been associated with a number of different cell functions. This includes participating in the 19S regulatory subunit of the proteasome [2], whose main function is degradation of excess, no longer needed, and defective proteins. In most organisms proteasome activity acts via the ubiquitin/26S proteasome system [3, 4]. This system involves the specific attachment of a chain of ubiquitin to the protein target by the E1-E3 enzymes [3]. The 265 proteasome complex recognizes labeled target proteins. This complex can be subdivided into a $20 \mathrm{~S}$ core protease and a $19 \mathrm{~S}$ regulatory part [5]. The $19 \mathrm{~S}$ subunit works by recognizing the target proteins and delivering them to the $20 \mathrm{~S}$ subunit for degradation. Among other proteins the $19 \mathrm{~S}$ subunit is composed by the AAA-ATPases Psmc 1-5 (Rpt1-Rpt6) (regulatory particle triple A ATPase) [6]. All Psmc/Rpt proteins are essential in yeast [7] and they form an hexameric ATPase complex [8, 9]. In Arabidopsis, mutants affecting 19S RP ATPase subunits show severe defects in maintaining the pool of stem cells in the root. Importantly, gametophyte development requires proteasome function, which is evident by chemical inhibition of the proteasome resulting in pollen developmental defects [10-12]. Recent studies using insertion mutants affecting proteasome components observed that alleles affecting Rpt5a (an Arabidopsis ortholog of Psmc3) displayed severe male gametophyte development defects, with pollen development arrested before cells enter meiosis at the second pollen mitosis stage [13].

PSMC3 has also being implicated in different cellular events that do not require proteolysis such as transcriptional initiation and elongation [14-16], DNA repair [17], and as a negative regulator of cell proliferation [18, 19]. By comparing gene expression profiles from normal and abnormal human testes with those from comparable infertile mouse models, a 
number of genes critical for male fertility have been identified [20]. Among the expression of 19 human genes that were different between normal and abnormal samples, Psmc3 appears as a top candidate [20]. Another lead to the function of PSMC3 independent of the proteasome is that the HOP2/TBPIP protein, a strong interaction partner of PSMC3, has an extensively documented role in proper meiotic chromosome segregation and fertility through its interaction with DMC1 and RAD51, both central components of the recombination pathway [21-24].

The role of PSMC3 in mammalian spermatogenesis has not been explored. Herein, we present the phenotype associated to the conditional deletion of Psmc3 in mouse gonads. Males are infertile likely a result of absence of any gametocyte type in the gonad. Meiosis is apparently not affected in $\mathrm{Psmc}^{-/-}$mice when ablation of PSMC3 occurs at meiotic stages. However, testis development is impaired at early stages during spermatogonia niche establishment, with massive loss of undifferentiated spermatogonia. Our work in understanding the functions of PSMC3 in germ cell development has broader implications in defining mechanisms responsible for infertility.

\section{Materials and Methods}

\section{Mice and Genotyping}

Experiments involving mice conformed to relevant regulatory standards and were approved by the IACUC (Institutional Animal Care and Use Committee).

Mice: The Psmc3 stem cells carrying a floxed allele mice was obtained from International Knockout Mouse Consortium. Transgenic Cre recombinase mice Ddx4-Cre FVB-Tg(Ddx4-cre)1Dcas/J $^{\text {or }}$ Stra8-iCre ${ }^{\text {B6.FVB-Tg(Stra8-icre)1Reb/LguJ }}$ were purchased from The Jackson Laboratory (Bar Harbor, ME). Spo11-Cre mice were provided by Dr. P. Jordan (Johns Hopkins University Bloomberg School of Public Health, Baltimore, MD). All mice were maintained on a mixed genetic 
background at the Laboratory Animal Resource Center of Oklahoma Medical Research Foundation. All animal work was carried out in accordance with IACUC protocols.

Genotyping: characterization of wild type and floxed alleles was carried out by PCR using the following oligonucleotides (see Fig. 2A): 1F 5'- CAAGCAGATCCAGGAGGTAAG, 1R 5'-

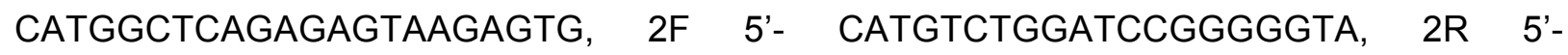
CCTACTGCGACTATAGAGATATC, 3F 5'- GGATTCCAGAGAGATTGGAGATTGT, 3R 5'CCTACTGCGACTATAGAGATATC, 4F 5'- GGATTCCAGAGAGATTGGAGATTGT, and 4R 5'GAACGGGCCACACAAATCTAGTA. The presence of cre recombinase allele was determine by PCR using the following primers: Spo11-Cre forward 5'-CCATCTGCCACCAGCCAG, Spo11-Cre reverse 5'-TCGCCATCTTCCAGCAGG,

\section{Histology and immunostaining}

Testes and ovaries were dissected, fixed in $4 \%$ paraformaldehyde and processed for paraffin embedding. After sectioning $(5-8-\mu \mathrm{m})$, tissues were positioned on microscope slides and analyzed using hematoxylin and eosin using standard protocols. For immunostaining analysis, tissue sections were deparaffinized, rehydrated and antigen was recovered in sodium citrate buffer (10 mM Sodium citrate, $0.05 \%$ Tween $20, \mathrm{pH}$ 6.0) by heat/pressure-induced epitope retrieval. Incubations with primary antibodies were carried out for $2 \mathrm{PLZF}$ at $37^{\circ} \mathrm{C}$ in $\mathrm{PBS} / \mathrm{BSA}$ $3 \%$. Primary antibodies used in this study were as follows: monoclonal mouse antibody raised against mouse SOX9 at 1:500 dilution (AbCam, ab26414), polyclonal rabbit antibody raised against mouse STRA8 at 1:500 dilution (AbCam, ab49602), polyclonal rabbit antibody raised 
against mouse TRA98 at 1:200 dilution (AbCam, 82527), monoclonal mouse antibody raised against mouse PLZF at 1:50 dilution (Santa Cruz, 28319). Following three washes in 1× PBS, slides were incubated for 1 PLZF at room temperature with secondary antibodies. A combination of Fluorescein isothiocyanate (FITC)-conjugated goat anti-rabbit IgG (Jackson laboratories) with Rhodamine-conjugated goat anti-mouse IgG and Cy5-conjugated goat antihuman $\lg$ each diluted 1:450 were used for simultaneous triple immunolabeling. For Stra8, we used the ImmPRESS ${ }^{\mathrm{TM}}$ Reagent Anti-Rabbit IgG Peroxidase (Vector Laboratories) and hematoxylin as counterstaining. Slides were subsequently counterstained for $3 \mathrm{~min}$ with $2 \mu \mathrm{g} / \mathrm{ml}$ DAPI containing Vectashield mounting solution (Vector Laboratories) and sealed with nail varnish.

\section{Statistical Analyses}

Results are presented as mean \pm standard deviation (SD). Statistical analysis was performed comparisons between 2 groups. $\mathrm{P}<0.05$ was considered statistically significant.

\section{Results}

\section{Psmc3 expression in mouse testis}

Tissue specific expression and kinetics of expression in testis may help reveal the function/s of PSMC3. We performed RT-PCR on total purified RNA from different mouse tissues and specific primers designed to analyze the level of expression of Psmc3 (Figure 1A). Our results are consistent with previous reports [25] and show that Psmc3 is highly expressed in testis and a relative minor amount in other tissues such as thymus, brain, liver, and kidney. 
We then used a complete data set of gene expression previously generated in testis [26] to determine Psmc3 expression during testis development (Figure 1B). We used Dmc1 and Hop2 expression times as markers for proteins expressed during early meiotic specific stages of gamete development. We concluded that Psmc3 is initially expressed at pre-meiotic stages of gamete development (6 days post partum (dpp) samples) and gradually increases after $10 \mathrm{dpp}$ as spermatogenesis progresses, which may suggest a dual role for Psmc3 both at early and later stages of gamete development.

\section{Generation of Psmc3 testis specific knockout mice}

We generated Psmc3 knockout mice using germline conditional inactivation. Two FRT sites are located in between exons 6 and 7 and flank LacZ, a loxP site, and a Neo cassette. Exons 7 deletion of the floxed allele only in cells already committed to meiosis (Stra8-Cre; Psmc3 ${ }^{\text {fl/ }}$,

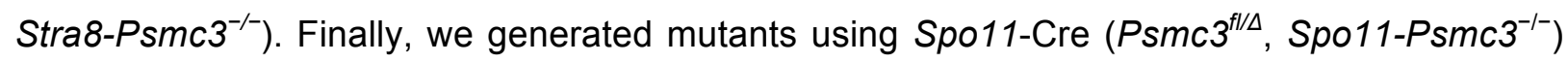
mice in which the floxed Psmc3 allele is expected to be deleted only in early primary spermatocytes. We confirmed deletion of Psmc3 by RT-qPCR (Fig. 2B).

\section{Deletion of Psmc3 results in testis developmental defects}

If Psmc3 participates in any stage of gamete development, we expect that their deletion will lead to disruption of gametogenesis, which can be studied by comparative tissue analysis of 
wild type and mutant testis. $P s m c 3^{-/}$adult mice appear normal in all aspects except in reproductive tissues (Fig. 2C). However, Stra8-Psmc $3^{-1-}(0.045 \mathrm{~g} \pm 0.005, \mathrm{n}=4, \mathrm{P}=0.0002, \mathrm{t}$ test) and $D d x 4-P s m c 3^{-/-}$males $(0.023 g \pm 0.003, n=3, P \leq 0.0001, t$ test $)$ had significantly smaller testes than wild type $(0.11 \mathrm{~g} \pm 0.003, \mathrm{n}=3)$ littermates, with $D d x 4-P s m c 3^{-/-}$showing the most significant reduction in testis size (Fig. 2C). This substantial reduction in size is an

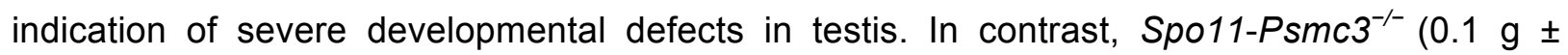
$0.0063, \mathrm{n}=4, \mathrm{P}=0.1, \mathrm{t}$ test) did not show any significant difference compared to wild type littermates (Fig. 2C). Spo11 is expressed in early prophase I, during leptonema [27]. Therefore, normal testis size in $\mathrm{Psmc}^{-/-}$with Spo11-Cre background indicates that developmental defects triggered by PSMC3 depletion are originated in pre-meiotic stages of gamete development and that PSMC3 has no apparent role during mouse meiosis. Thus, homozygous Stra8- and Ddx4$\mathrm{Psmc}^{-/-}$mutant mice show severe blocks of spermatogenesis. We also analyzed hematoxylineosin histological sections of 45-days-old wild type and $D d x 4-P s m c 3^{-/-}$ovaries. We note that albeit a significant reduction occurred in ovary size and increase in stromal cells, a reduced number of follicles can be observed in the $D d x 4-P s m c 3^{-/-}$mice (Fig. 2D). We conclude that Psmc3 plays a role in male and female gametogenesis. testicular hypoplasia with hyperplasia of interstitial cells and a lack of spermatozoa, with Ddx4$\mathrm{Psmc3}^{-/-}$showing the most severe phenotype (Fig. 3A and B). Although there were no alterations in number of seminiferous tubules, the diameter of seminiferous tubules was reduced (wild type, $319.8 \mu \mathrm{m} \pm 13.26, \mathrm{n}=30$; mutant, $173.6 \mu \mathrm{m} \pm 21.87, \mathrm{n}=30$ ). Spermatids represent the most advanced spermatogenic cells in the Stra8-Psmc $3^{-/-}$mice, indicating that spermatogenesis progress, albeit with a severely reduced number of cells (wild type average 53 cells per seminiferous tubules while Stra8-Psmc $3^{-/-}$average 32 cells per seminiferous 
tubule). Analysis of $D d x 4-P s m c 3^{-/-}$revealed near total loss of germ cells in seminiferous tubules. Although no meiocytes were observed, even those cell types at early development (i.e. spermatogonia); Sertoli cells were apparently not affected (Fig. 3A and B).

PSMC3 is apparently required for spermatogonia niche establishment and maintenance

The severe phenotype observed in $\mathrm{Ddx} 4-\mathrm{Psmc}^{-/-}$mice (Fig. 2 and $3 \mathrm{~A}$ and B) prompted us to explore earlier stages of testis development with the premise that morphological changes between the mutant and wild type may reveal differences in early spermatogenesis differentiation. Testis from mice at different postnatal ages were collected, paraffin embedded, and tissue slides analyzed by PLZF\&E or immunohistochemistry. PLZF\&E stained testis sections from 9dpp Ddx4-Psmc $3^{-/-}$show near absence of gametocyte and differences in cell composition compared to wild type (Fig. 3C). To analyze this in detail, we immunostained testis sections with Stra8, which marks differentiating spermatogonia (Fig. 3D). While several tubules in wild type contain cells expressing STRA8 (12.83 average number of cells per positive tubule, $\mathrm{n}=34$ seminiferous tubules), tubules in $\mathrm{Psmc}^{-/-}$samples show near absence of positive cells for these markers (0 STRA8 positive cells, $n=50$ ) (Fig. 3D). These results suggested that testis developmental defects in $P s m c 3^{-/-}$mice begin early during pre-meiotic stages of postnatal development, possibly before spermatogonia differentiate, and are the cause of absent germ cells in adult mutant mice.

To investigate this in detail, we then analyzed 1, 3, 5, 7 and 9 dpp testis sections by immunostaining with antibodies specific for PLZF/ZBTB16, used as a marker for undifferentiated spermatogonia, and TRA98, which mark all germ cells (Fig. 4A). Similar number of cells are positive for both markers as observed for both wild type and Psmc3 mutant in 1dpp (PLZF wild type, average \pm standard deviation, $1.0 \pm 1.3, n=123$ seminiferous tubules; mutant, $1.6 \pm 1.7, \mathrm{n}=73, \mathrm{P}=0.014$, $\mathrm{t}$ test) (TRA98 wild type, $0.33 \pm 0.73, \mathrm{n}=93$; mutant, $0.08 \pm 0.3, n=73, P=0.007$, t test) and 3dpp (PLZF wild type, $2.0 \pm 1.3, n=66$ 
seminiferous tubules; mutant, $2.0 \pm 1.6, \mathrm{n}=23, \mathrm{P}=0.89$, $\mathrm{t}$ test) (TRA98 wild type, $0.94 \pm 0.97$, $\mathrm{n}=66$; mutant, $1.4 \pm 1.02, \mathrm{n}=23, \mathrm{P}=0.05$, $\mathrm{t}$ test) testis.

Notably, compared to wild type (5dpp PLZF, $3.7 \pm 1.6, n=20$; TRA98, $3.9 \pm 1.8, n=26$.

7dpp PLZF, $3.8 \pm 2.3, n=45 ;$ TRA98, $3.1 \pm 1.5, n=48.9 \mathrm{dpp}$ PLZF, $6.0 \pm 3.5, \mathrm{n}=86 ;$ TRA98, $11.62 \pm 5.9, \mathrm{n}=86$ ) a significant reduction of PLZF and TRA98 positive cells in $\mathrm{Psmc}^{-/-}$ mutants were observed at 5ddp (PLZF, $0.0 \pm 0.0, n=20, P<0.0001, t$ test; TRA98, $0.0 \pm 0.0$, $\mathrm{n}=39, \mathrm{P}<0.0001, \mathrm{t}$ test $)$ and $7 \mathrm{dpp}(\mathrm{PLZF}, 0.0 \pm 0.0, \mathrm{n}=41, \mathrm{P}<0.0001$, $\mathrm{t}$ test; TRA98, $0.0 \pm 0.0$, $\mathrm{n}=41, \mathrm{P}<0.0001, \mathrm{t}$ test $)$ and confirmed at 9dpp (PLZF, $0.0 \pm 0.0, \mathrm{n}=86, \mathrm{P}<0.0001, \mathrm{t}$ test; TRA98, $0.0 \pm 0.0, n=94, P<0.0001, t$ test) testis (Fig. 4A-C). We note that the reduction in the number of positive cells for PLZF indicates that deletion of Psmc3 affects undifferentiated stages of gamete development. 10.7, $\mathrm{n}=64$ and mutant $25.6 \pm 4.9, \mathrm{n}=75, \mathrm{P}=0.18$, t test) (Fig. 4A and $\mathrm{D}$ ), we conclude that deletion of Psmc3 by $D d x 4$-Cre affects germ cells at the undifferentiated stage of spermatogonia.

\section{Discussion}

PSMC3 has been associated with a number of different cell functions, and it is highly

expressed in testis. Nonetheless, PSMC3 function in gametogenesis is poorly understood. In this work, we took to task the function of PSMC3 in mouse gamete development. We 
We observed that knocking out Psmc3 in mouse spermatocytes results in severe male and female gonad developmental defect. Arrest of gametogenesis occurs at early pre-meiotic stages, revealed by a massive loss of undifferentiated spermatogonia, and apparently as a result of abnormal spermatogonia niche establishment and/or maintenance. Our results are in agreement with previous works showing that mutants affecting Rpt5a Arabidopsis (ortholog of Psmc3), result in severe male gametophyte defects, with pollen development arrested before cells enter meiosis at the second pollen mitosis stage. This correlates with absence of the proteasome-dependent cyclin A3 degradation and argues that gametophyte development may require proteasome function through RPR5A [13].

PSMC3 has also been associated to proteasome-independent functions. Indeed, PSMC3/TBPinteracts with HOP2/TBPIP [25], a central player in the meiotic recombination pathway. Deletion of HOP2 in mouse results in male and female gamete developmental defects, with impairment in double-strand break repair and homologous chromosome associations [28]. Because HOP2/TBPIP is a strong interactor of PSMC3, we reason that deletion of Psmc3, which may affect HOP2 integrity or activity, may result in meiotic defect. To test this, we analyzed Spo11-Psmc $3^{-/-}$mouse testis, in which conditional deletion of $P s m c 3$ is predicted to occur at the onset of primary spermatocytes, after normal mitotic divisions and developing gametes have entered meiosis. Evaluated by the normal development of Spo11$\mathrm{Psmc3}^{-/-}$testis, we conclude that PSMC3 is dispensable for normal meiotic progression, including double-stand break repair and homologous chromosome interactions.

In conclusion, our work defines a fundamental role of PSMC3 in spermatogenesis during early spermatogonia development. Future work, should address the mechanism of such function, either related or independent of PSMC3 participation in the proteasome. 


\section{References}

282 1. Mecklenburg JM, Hermann BP. Mechanisms Regulating Spermatogonial Differentiation. Results Probl

283

2842.

285

286

2873

2884.

289

2905.

291

2926.

293

2947.

295

296

297

298

2999.

300

301

302

303

304

305

306

307

308

309

310

311

312

313

314

315

316

317

318

319

320

321

322

323

324

325

326

327

328

32920.

330

Cell Differ 2016; 58:253-287.

Sepe M, Festa L, Tolino F, Bellucci L, Sisto L, Alfano D, Ragno P, Calabro V, de Franciscis V, La Mantia G, Pollice A. A regulatory mechanism involving TBP-1/Tat-Binding Protein 1 and Akt/PKB in the control of cell proliferation. PLoS One 2011; 6:e22800.

3. Hershko A, Ciechanover A. The ubiquitin system. Annu Rev Biochem 1998; 67:425-479.

4. Smalle J, Vierstra RD. The ubiquitin 26S proteasome proteolytic pathway. Annu Rev Plant Biol 2004; 55:555-590.

5. Walz J, Erdmann A, Kania M, Typke D, Koster AJ, Baumeister W. 26S proteasome structure revealed by three-dimensional electron microscopy. J Struct Biol 1998; 121:19-29.

6. Glickman MH, Rubin DM, Fried VA, Finley D. The regulatory particle of the Saccharomyces cerevisiae proteasome. Mol Cell Biol 1998; 18:3149-3162.

7. Rubin DM, Glickman MH, Larsen CN, Dhruvakumar S, Finley D. Active site mutants in the six regulatory particle ATPases reveal multiple roles for ATP in the proteasome. EMBO J 1998; 17:49094919.

8. Fu H, Reis N, Lee $\mathrm{Y}$, Glickman MH, Vierstra RD. Subunit interaction maps for the regulatory particle of the 26S proteasome and the COP9 signalosome. EMBO J 2001; 20:7096-7107.

9. Benaroudj N, Zwickl P, Seemuller E, Baumeister W, Goldberg AL. ATP hydrolysis by the proteasome regulatory complex PAN serves multiple functions in protein degradation. Mol Cell 2003; 11:69-78.

10. Speranza A, Scoccianti V, Crinelli R, Calzoni GL, Magnani M. Inhibition of proteasome activity strongly affects kiwifruit pollen germination. Involvement of the ubiquitin/proteasome pathway as a major regulator. Plant Physiol 2001; 126:1150-1161.

11. Sheng X, Hu Z, Lu H, Wang X, Baluska F, Samaj J, Lin J. Roles of the ubiquitin/proteasome pathway in pollen tube growth with emphasis on MG132-induced alterations in ultrastructure, cytoskeleton, and cell wall components. Plant Physiol 2006; 141:1578-1590.

12. Doelling JH, Yan N, Kurepa J, Walker J, Vierstra RD. The ubiquitin-specific protease UBP14 is essential for early embryo development in Arabidopsis thaliana. Plant J 2001; 27:393-405.

13. Gallois JL, Guyon-Debast A, Lecureuil A, Vezon D, Carpentier V, Bonhomme S, Guerche P. The Arabidopsis proteasome RPT5 subunits are essential for gametophyte development and show accession-dependent redundancy. Plant Cell 2009; 21:442-459.

14. Lassot I, Latreille D, Rousset E, Sourisseau M, Linares LK, Chable-Bessia C, Coux O, Benkirane M, Kiernan RE. The proteasome regulates HIV-1 transcription by both proteolytic and nonproteolytic mechanisms. Mol Cell 2007; 25:369-383.

15. Lee D, Ezhkova E, Li B, Pattenden SG, Tansey WP, Workman JL. The proteasome regulatory particle alters the SAGA coactivator to enhance its interactions with transcriptional activators. Cell 2005; 123:423-436.

16. Gonzalez F, Delahodde A, Kodadek T, Johnston SA. Recruitment of a 19S proteasome subcomplex to an activated promoter. Science 2002; 296:548-550.

17. Russell SJ, Reed SH, Huang W, Friedberg EC, Johnston SA. The 19S regulatory complex of the proteasome functions independently of proteolysis in nucleotide excision repair. Mol Cell 1999; 3:687695.

18. Park BW, O'Rourke DM, Wang Q, Davis JG, Post A, Qian X, Greene MI. Induction of the Tat-binding protein 1 gene accompanies the disabling of oncogenic erbB receptor tyrosine kinases. Proc Natl Acad Sci U S A 1999; 96:6434-6438.

19. Pollice A, Nasti V, Ronca R, Vivo M, Lo lacono M, Calogero R, Calabro V, La Mantia G. Functional and physical interaction of the human ARF tumor suppressor with Tat-binding protein-1. J Biol Chem 2004; 279:6345-6353.

20. Rockett JC, Patrizio P, Schmid JE, Hecht NB, Dix DJ. Gene expression patterns associated with infertility in humans and rodent models. Mutat Res 2004; 549:225-240. 
331 21. Petukhova GV, Pezza RJ, Vanevski F, Ploquin M, Masson JY, Camerini-Otero RD. The Hop2 and

332

333

334

335

336

337

338

339

340

341

342

343

344

345

346

347

348

349

350

351

352

353

354

355

356

357

358

359

360

361

362

363

364

365 Mnd1 proteins act in concert with Rad51 and Dmc1 in meiotic recombination. Nat Struct Mol Biol 2005; 12:449-453.

22. Pezza RJ, Petukhova GV, Ghirlando R, Camerini-Otero RD. Molecular activities of meiosis-specific proteins Hop2, Mnd1, and the Hop2-Mnd1 complex. J Biol Chem 2006; 281:18426-18434.

23. Pezza RJ, Voloshin ON, Vanevski F, Camerini-Otero RD. Hop2/Mnd1 acts on two critical steps in Dmc1-promoted homologous pairing. Genes Dev 2007; 21:1758-1766.

24. Pezza RJ, Voloshin ON, Volodin AA, Boateng KA, Bellani MA, Mazin AV, Camerini-Otero RD. The dual role of HOP2 in mammalian meiotic homologous recombination. Nucleic Acids Res 2014; 42:23462357.

25. Satoh T, Ishizuka T, Tomaru T, Yoshino S, Nakajima Y, Hashimoto K, Shibusawa N, Monden T, Yamada M, Mori M. Tat-binding protein-1 (TBP-1), an ATPase of 19S regulatory particles of the 26S proteasome, enhances androgen receptor function in cooperation with TBP-1-interacting protein/Hop2. Endocrinology 2009; 150:3283-3290.

26. Margolin G, Khil PP, Kim J, Bellani MA, Camerini-Otero RD. Integrated transcriptome analysis of mouse spermatogenesis. BMC Genomics 2014; 15:39.

27. Bellani MA, Boateng KA, McLeod D, Camerini-Otero RD. The expression profile of the major mouse SPO11 isoforms indicates that SPO11beta introduces double strand breaks and suggests that SPO11alpha has an additional role in prophase in both spermatocytes and oocytes. Molecular and Cellular Biology 2010; 30:4391-4403.

28. Petukhova GV, Romanienko PJ, Camerini-Otero RD. The Hop2 protein has a direct role in promoting interhomolog interactions during mouse meiosis. Dev Cell 2003; 5:927-936.

\section{Figure legends}

Figure 1. Psmc3 expression during gametogenesis. (A) Expression of Psmc3 in different tissues assessed by RT-PCR (B) Kinetics of Psmc3 gene expression in testis of 6, 10, 12, 14, 16, 18, 20, and $38 \mathrm{dpp}$ mice. Expression of Psmc3 and Dmc1 and Hop2 was assessed by RNAseq.

Figure 2. Psmc3 gene targeting design and testis size phenotype of Psmc3 mutant mice.

(A) Testis specific Cre knockout strategy for deletion of Psmc3. A trapping cassette was inserted to delete exons 7 and 8 in Psmc3. (B) Psmc3 transcription levels expression in whole testis of wild type and mutant $\left(P s m c 3^{-/}\right.$, Spo11-Cre $)$mice and enriched fractions of spermatogonia cells from wild type and mutant $\left(P s m c 3^{-/}, D d x 4-C r e\right)$ mice assessed by RTPCR. (C) PLZF\&E stained testis of wild type, Stra8-Psmc $3^{-/-}, D d x 4-P s m c 3^{-/}$, and Spo11- 
$\mathrm{Psmc3}^{-/-}$mice. Quantification of testis weight for wild type and homozygous knockout mice is also shown. (D) PLZF\&E stained ovaries of wild type and $D d x 4-P s m c 3^{-/-}$mice.

Figure 3. Ddx4-Psmc $3^{-/-}$mice show profound defects in gametogenesis. (A) Details of histological sections stained with PLZF\&E of wild type, Stra8-Psmc $3^{-/}$, and $D d x 4-P s m c 3^{-/-}$ seminiferous tubules. Stars mark seminiferous tubules with absent germ cells. Note unchanged number and morphology of Sertoli cells (indicated by green arrows). (B) Histological sections of wild type and Ddx4-Psmc $3^{-/-}$testis immunostained with SOX9 (to mark Sertoli cells) and DAPI (to mark nuclei). (C) PLZF\&E stained 9dpp testis of wild type and Ddx4-Psmc ${ }^{-/-}$mice. The inserts show magnification of seminiferous tubules and composition and distribution of cells. (D) STRA8 immunostained and hematoxylin stained 9dpp testis of wild type and Ddx4-Psmc $3^{-/-}$ mice. Starts indicate positive seminiferous tubules. Quantitation of number of cells STRA8 positive per positive seminiferous tubule is also shown. antibodies (marking germ cells), PLZF antibodies (marking undifferentiated spermatogonia) 
Figure 1
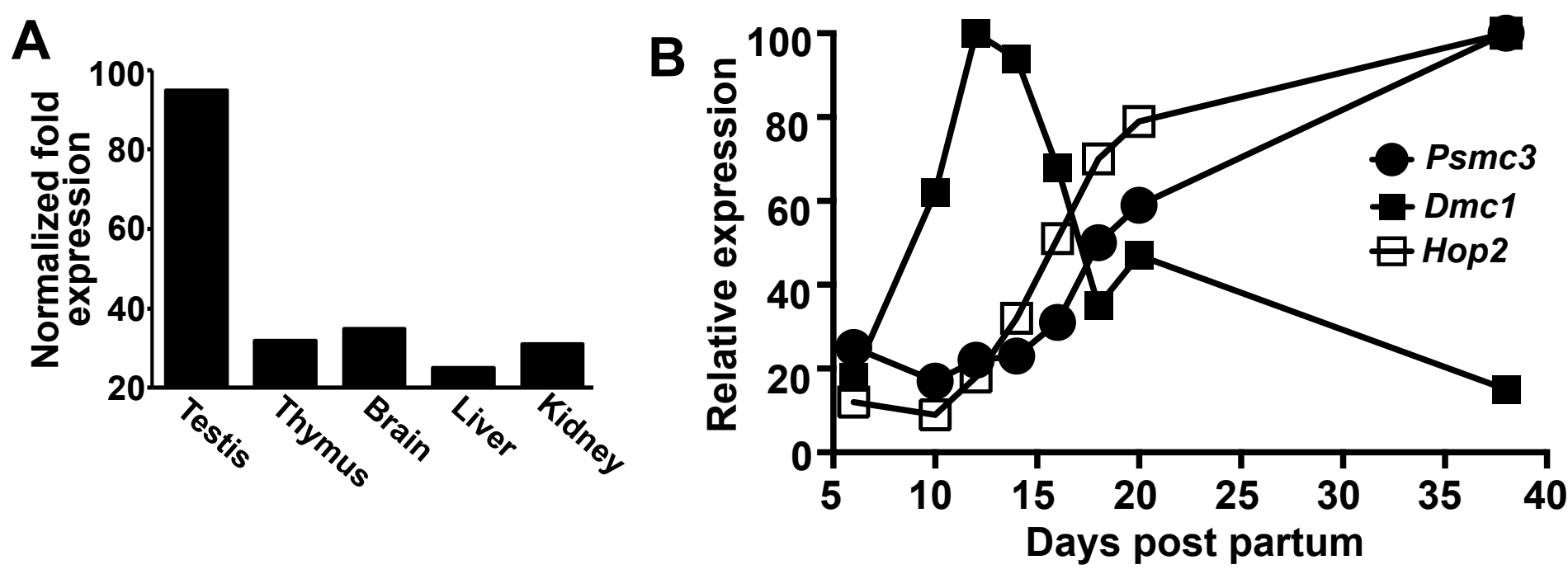
Figure 2

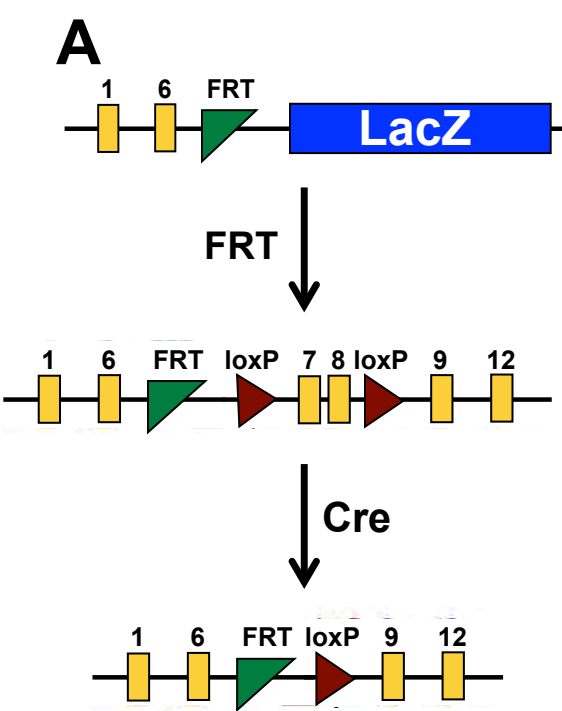

D Wild type

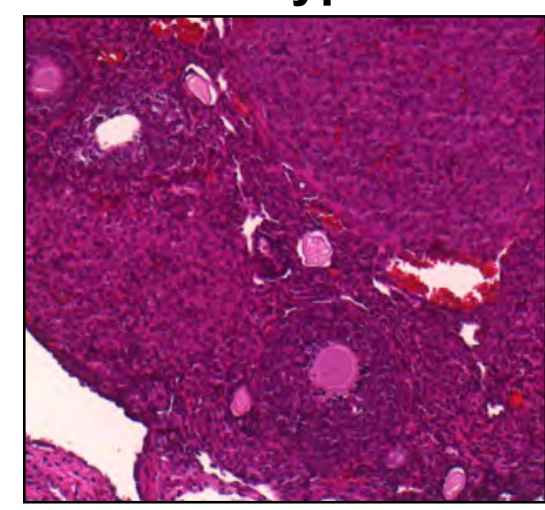

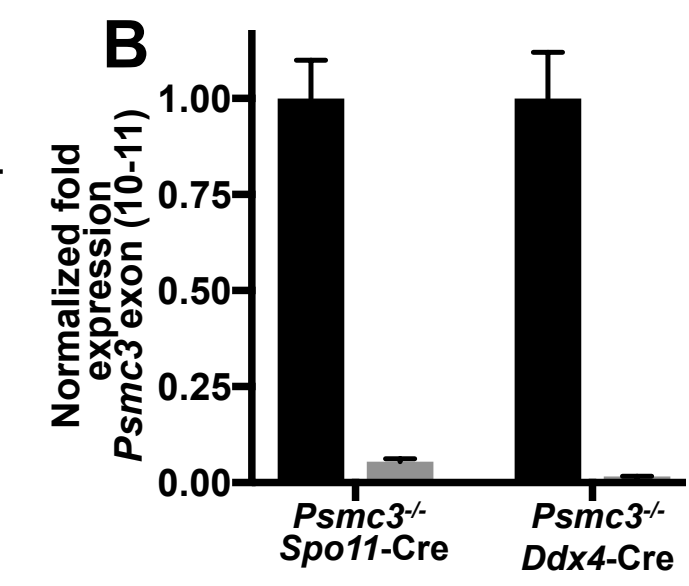

C

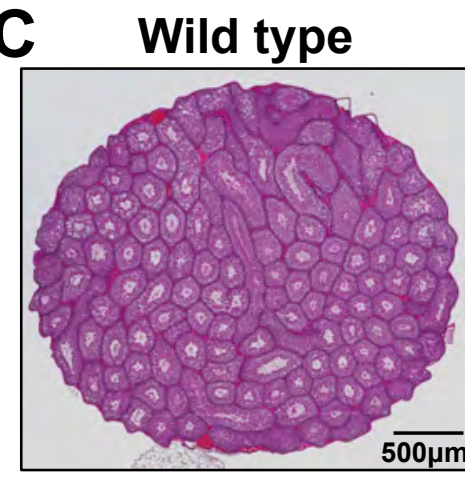

Psmc3--- Ddx4-Cre

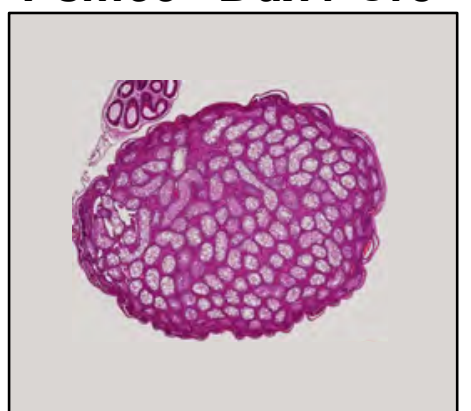

Psmc3\%- Ddx4-Cre

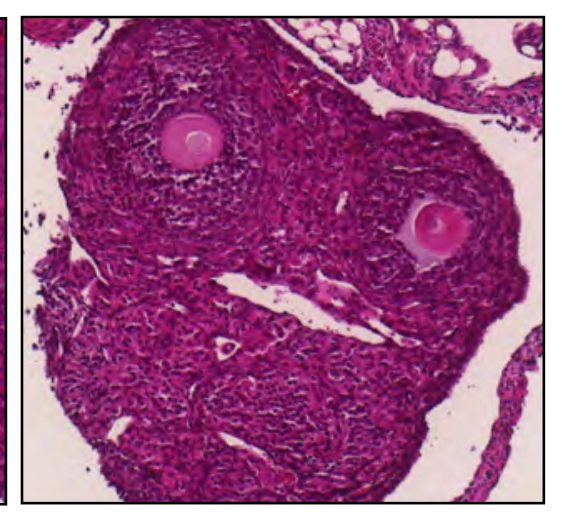

Psmc3 ${ }^{-/-}$Stra8-Cre

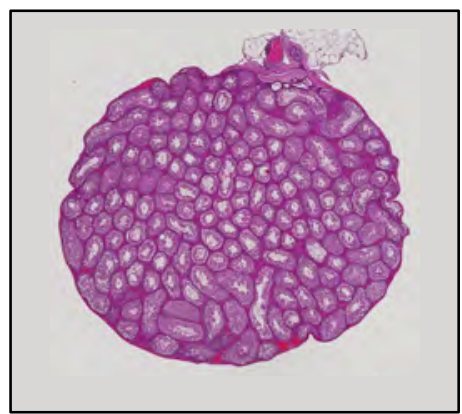

Psmc3-/-Spo11-Cre
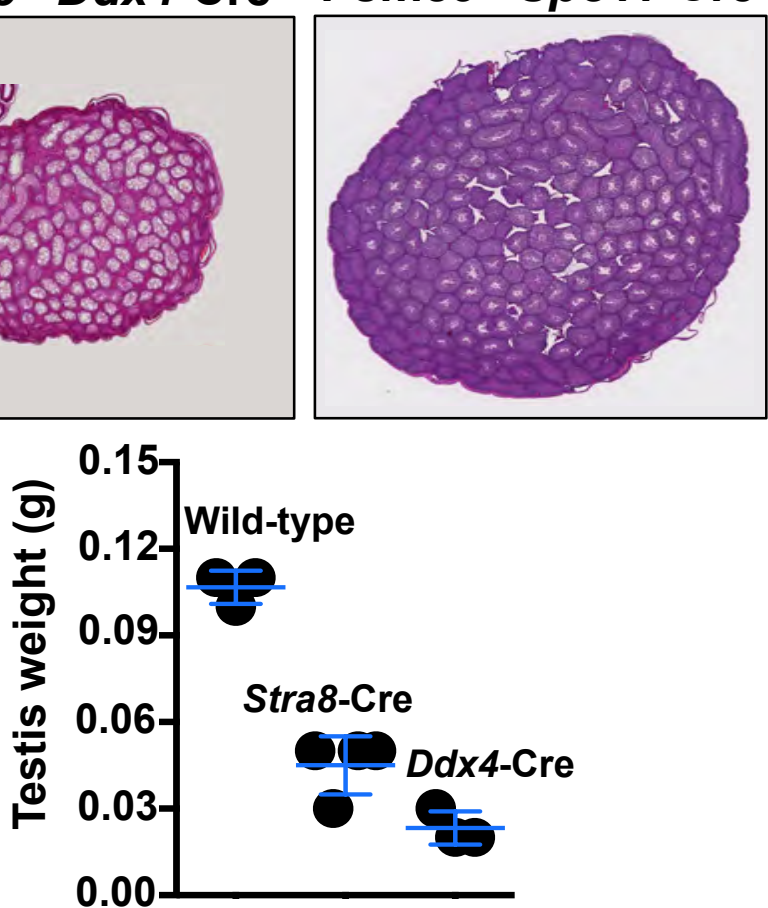

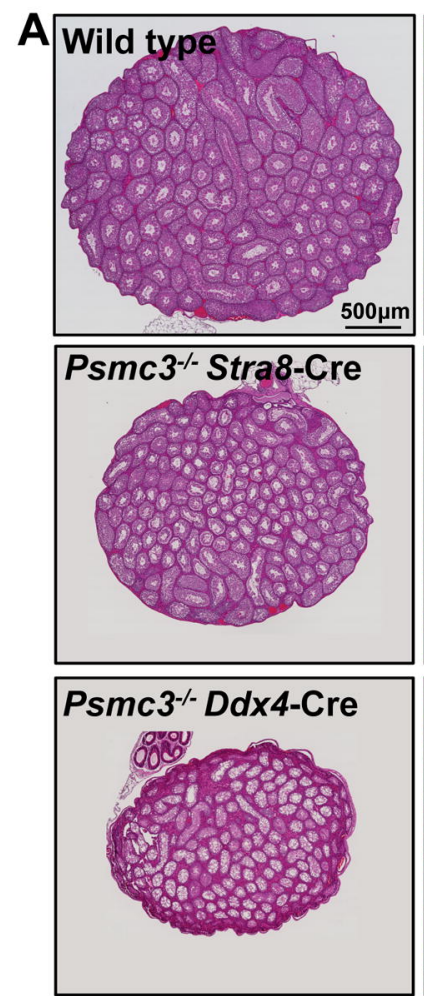
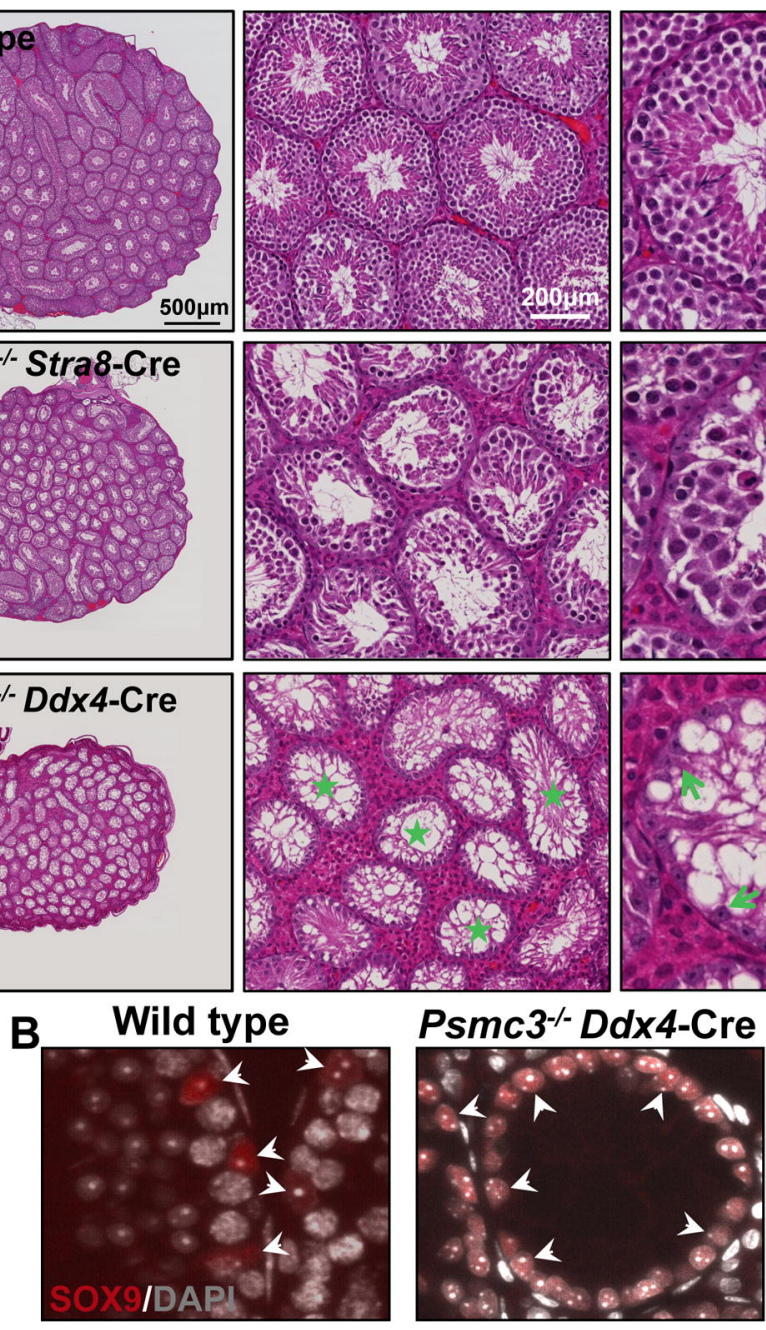

C
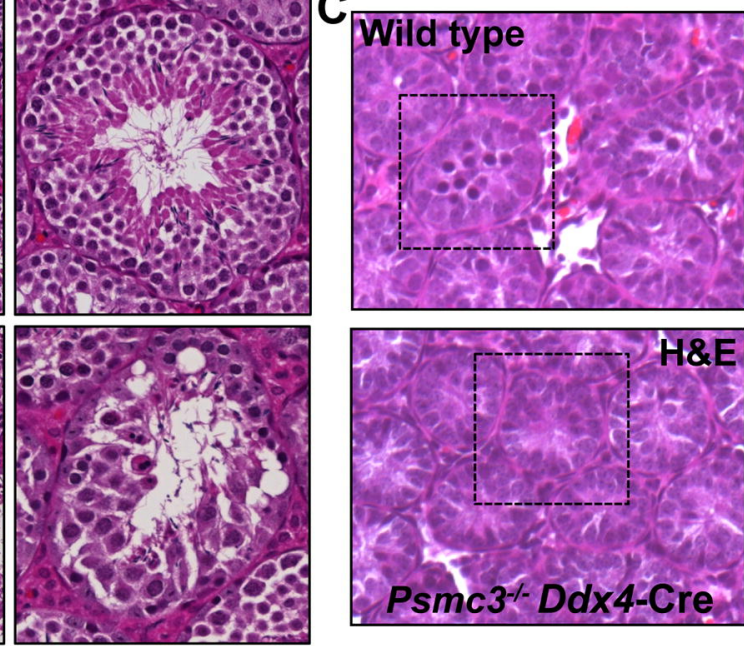

Germ cells Sertoli cells
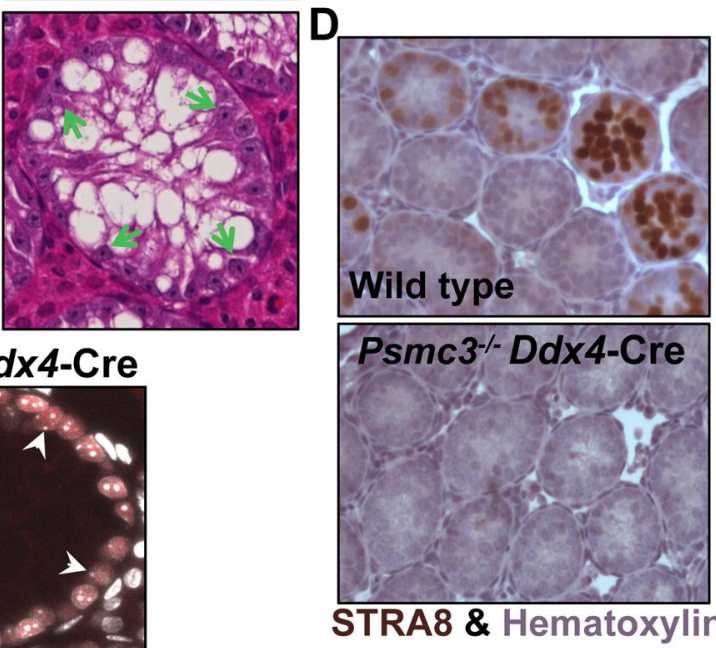

Figure 3
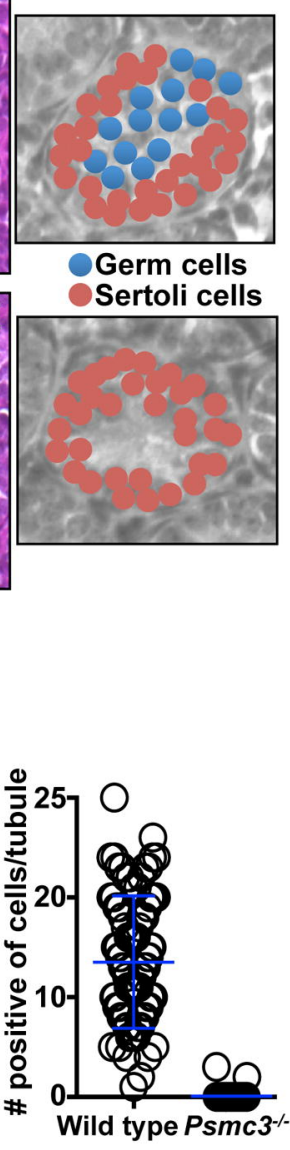

STRA8 \& Hematoxylin 


\section{A Wild type}

\section{음}

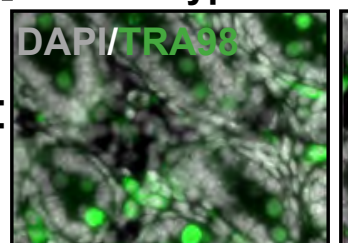

응
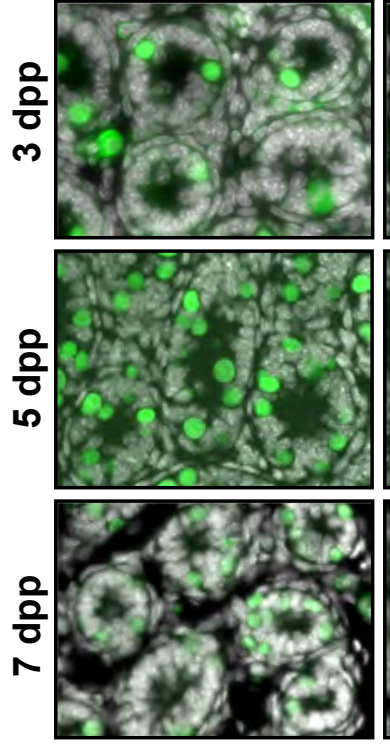

$\frac{8}{q}$

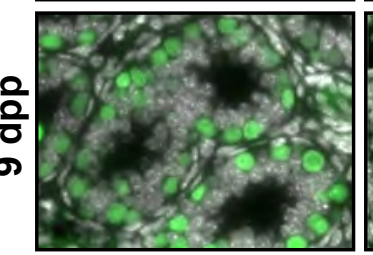

$1 \mathrm{dpp}$

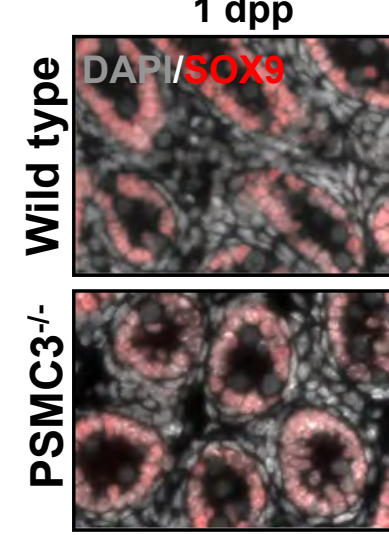

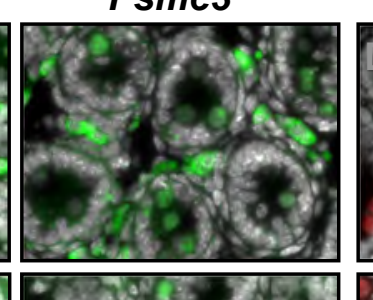
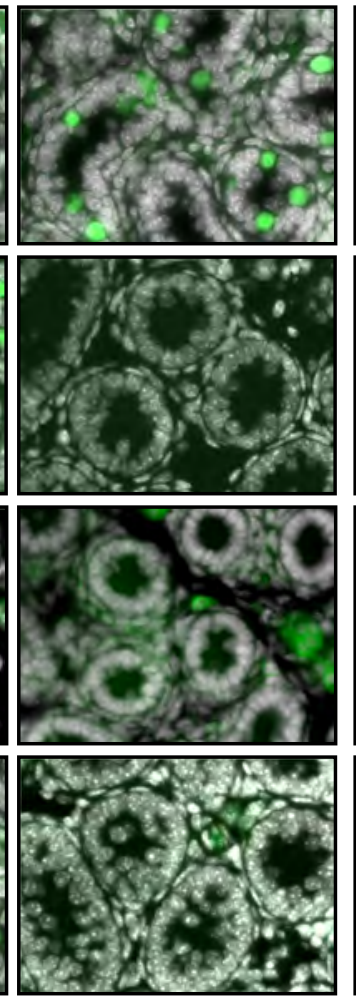

3 dpp

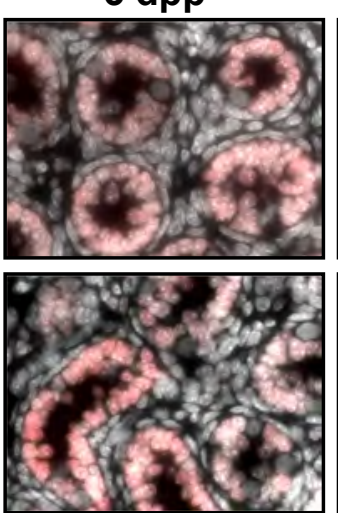

Wild type
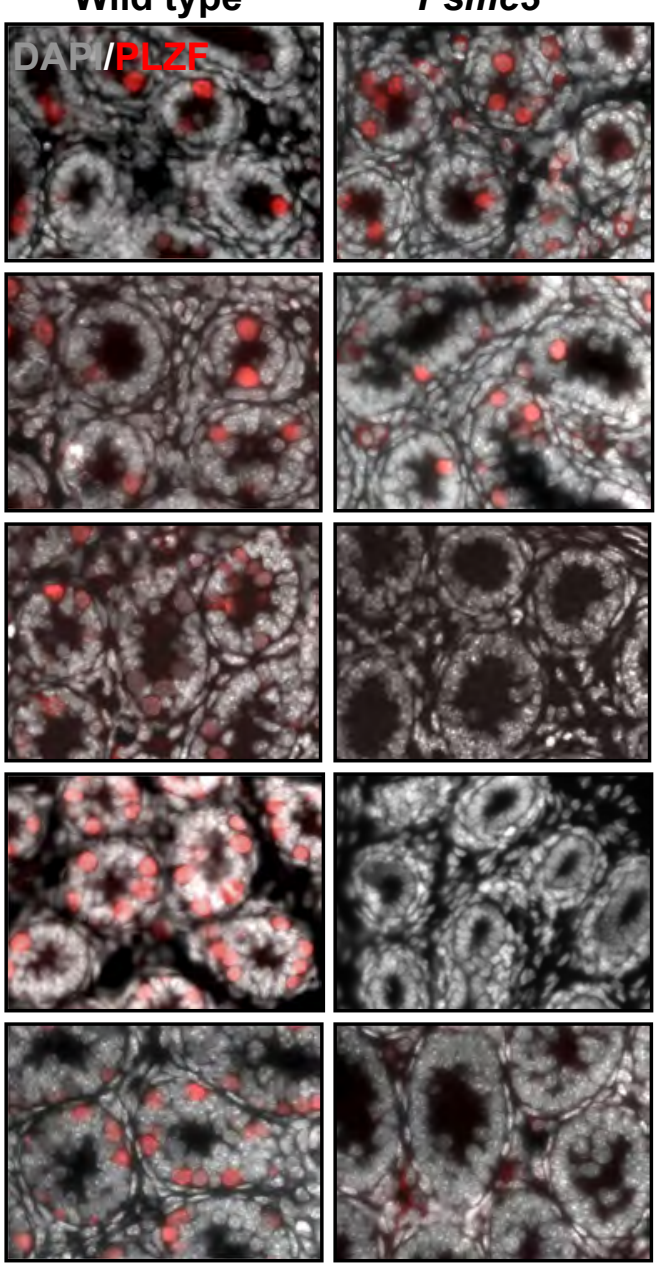

5 dpp

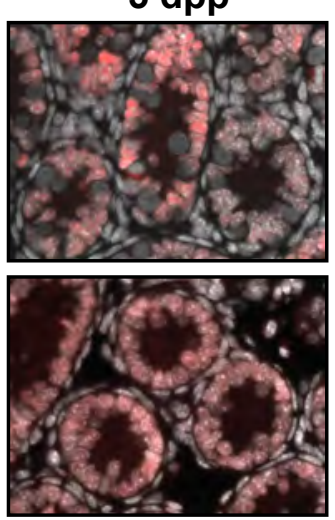

$9 \mathrm{dpp}$

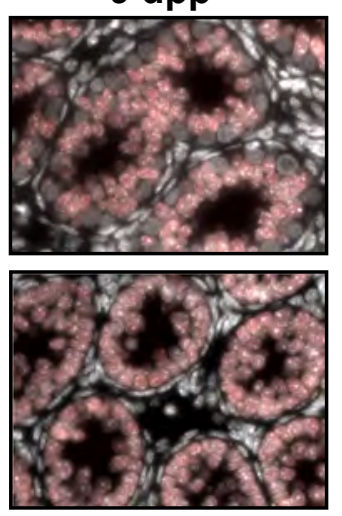

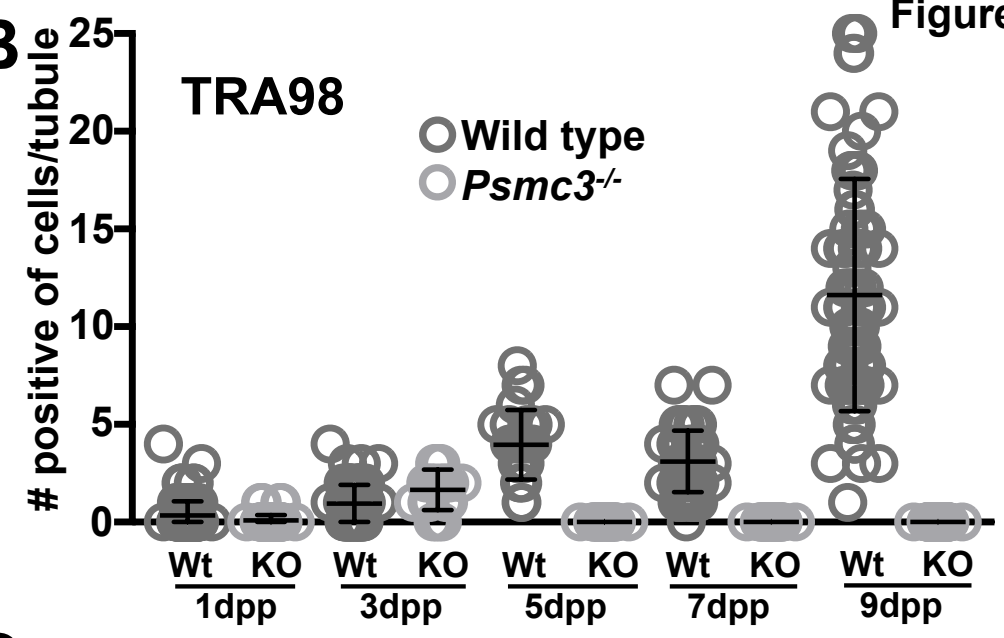

C

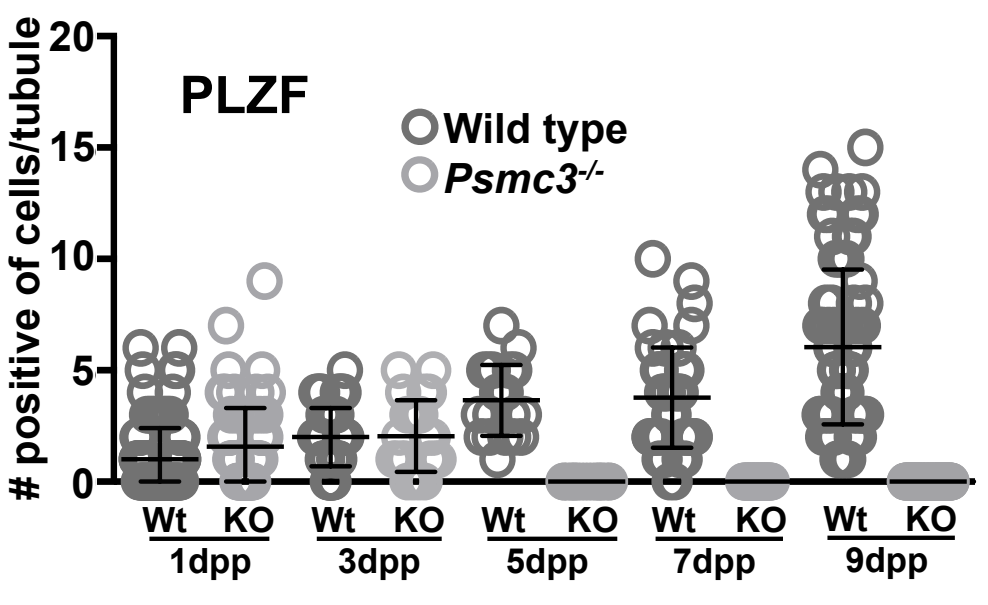

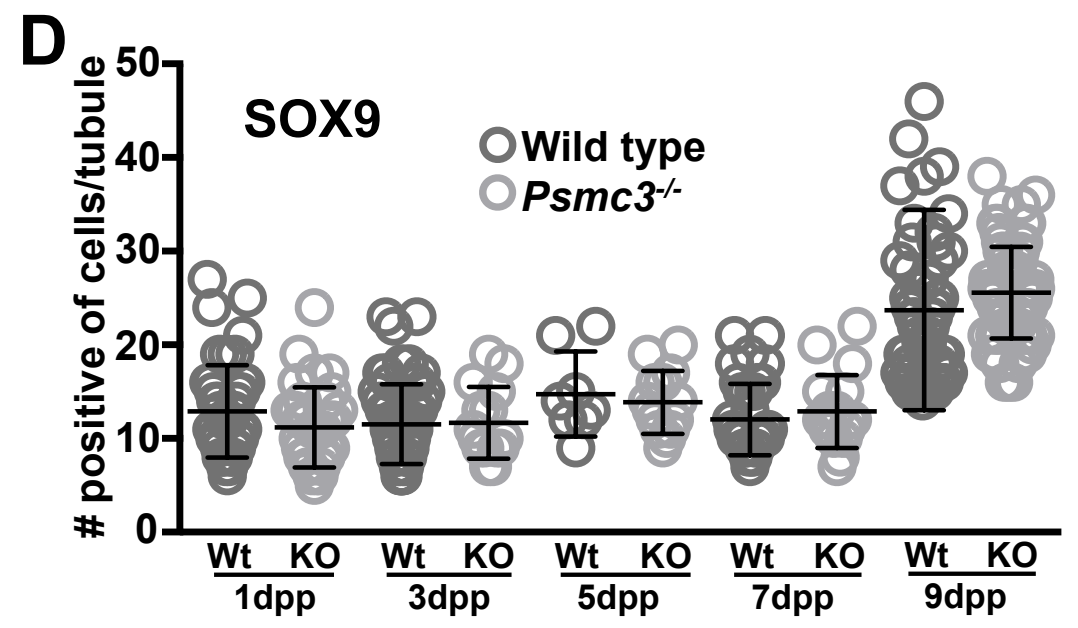

